\title{
BOUNDEDNESS OF SUBLINEAR OPERATORS ON THE HOMOGENEOUS HERZ SPACES
}

\author{
Guoen Hu
}

\begin{abstract}
Some boundedness results are established for sublinear operators on the homogeneous Herz spaces. As applications, some new theorems about the boundedness on homogeneous Herz spaces for commutators of singular integral operators are obtained.
\end{abstract}

\section{Introduction}

We will work on $\mathbb{R}^{n}, n \geq 1$. Let $K(x)$ be a function on $\mathbb{R}^{n} \backslash\{0\}$ which satisfies the size condition

$$
|K(x)| \leq C|x|^{-n}, \quad x \in \mathbb{R}^{n} \backslash\{0\} .
$$

A celebrated result of Stein [15] tells us that if the operator

$$
T f(x)=\text { p.v. } \int_{\mathbb{R}^{n}} K(x-y) f(y) d y
$$

is bounded on $L^{q}\left(\mathbb{R}^{n}\right)$ for some $q>1$, then $T$ is also bounded on $L^{q}\left(\mathbb{R}^{n},|x|^{\alpha} d x\right)$ provided that $-n<\alpha<n(q-1)$, where $L^{q}\left(\mathbb{R}^{n},|x|^{\alpha} d x\right)$ denotes the weighted Lebesgue space defined by

$$
\begin{aligned}
& L^{q}\left(\mathbb{R}^{n},|x|^{\alpha} d x\right)=\left\{f \text { is measurable on } \mathbb{R}^{n}\right. \text { and } \\
& \left.\qquad\|f\|_{L^{q}\left(\mathbb{R}^{n},|x|^{\alpha} d x\right)}=\int_{\mathbb{R}^{n}}|f(x)|^{q}|x|^{\alpha} d x<\infty\right\} .
\end{aligned}
$$

Soria and Weiss [14] gave some beautiful generalizations of Stein's result. In particular, they obtained the result of Stein in the case $q=1$.

2000 Mathematics Subject Classification. 42B20.

Key words. Sublinear operator, homogeneous Herz space, power weight, commutator, $\operatorname{BMO}\left(\mathbb{R}^{n}\right)$.

The research was supported by the NSF of Henan Province. 
Yang and some other authors (see $[\mathbf{1 0}],[\mathbf{1 1}]$ and $[\mathbf{1 2}]$ ) considered the boundedness of sublinear operators on homogeneous Herz spaces. Since the homogeneous Herz spaces are some kind of generalization of the weighted Lebesgue spaces with power weights, these results are of great interest. Lu and Yang [12], Hu, Lu and Yang [10] considered the boundedness on homogeneous Herz spaces for commutators and obtained many useful theorems. But in some interesting cases, the methods and results in $[\mathbf{1 2}]$ and $[\mathbf{1 0}]$ break down. Let us consider the commutator of singular integral operator defined by

$$
T_{b, m} f(x)=\int_{\mathbb{R}^{n}}(b(x)-b(y))^{m} \frac{\Omega(x-y)}{|x-y|^{n}} f(y) d y,
$$

where $\Omega$ is homogeneous of degree zero and has mean value zero on the unit sphere $S^{n-1}, m$ is a positive integer and $b \in \mathrm{BMO}\left(\mathbb{R}^{n}\right)$. If $\Omega \in L^{q}\left(S^{n-1}\right)$ for some $q>1$, we can deduce the boundedness on Herz space for $T_{b, m}$ by employing the results of [12] and [10]. Although it was proved in [9] that $\Omega \in L(\log L)^{m+1}\left(S^{n-1}\right)$ (i.e. $\int_{S^{n-1}}|\Omega(x)| \log ^{m+1}(2+$ $|\Omega(x)|) d x<\infty)$ is a sufficient condition such that the operator $T_{b, m}$ defined by (1) is bounded on $L^{p}\left(\mathbb{R}^{n}\right)$ with bound $C(n, m, p)\|b\|_{\mathrm{BMO}\left(\mathbb{R}^{n}\right)}^{m}$ for all $1<p<\infty$, we do not know the behaviour on homogeneous Herz spaces, even on weighted Lebesgue spaces with power weights, for $T_{b, m}$ when $\Omega \notin \cup_{q>1} L^{q}\left(S^{n-1}\right)$. The main purpose of this paper is to establish some boundedness results on the homogeneous Herz spaces for sublinear operators which are particularly suitable for commutators of singular integral operators and some other important operators. To state our results, let us recall the definition of the homogeneous Herz spaces.

Let $C_{k}=\left\{x \in \mathbb{R}^{n}: 2^{k-1}<|x| \leq 2^{k}\right\}$ for $k \in \mathbb{Z}$. For a measurable set $E \subset \mathbb{R}^{n}$, denote by $\chi_{E}$ the characteristic function of $E$. Set $\chi_{k}=\chi_{C_{k}}$.

Definition. Let $\alpha \in \mathbb{R}, 0<p, q \leq \infty$. The homogeneous Herz space $\dot{K}_{q}^{\alpha, p}\left(\mathbb{R}^{n}\right)$ is defined by

$$
\dot{K}_{q}^{\alpha, p}\left(\mathbb{R}^{n}\right)=\left\{f \in L_{\mathrm{loc}}^{q}\left(\mathbb{R}^{n} \backslash\{0\}\right):\|f\|_{\dot{K}_{q}^{\alpha, p}\left(\mathbb{R}^{n}\right)}<\infty\right\},
$$

where

$$
\|f\|_{\dot{K}_{q}^{\alpha, p}\left(\mathbb{R}^{n}\right)}=\left\{\sum_{k=-\infty}^{\infty} 2^{k \alpha p}\left\|f \chi_{k}\right\|_{L^{q}\left(\mathbb{R}^{n}\right)}^{p}\right\}^{1 / p}
$$

with the usual modification made when $p=\infty$ or $q=\infty$. 
For the properties and applications of the space $\dot{K}_{q}^{\alpha, p}\left(\mathbb{R}^{n}\right)$, and the boundedness of some classical operators on $\dot{K}_{q}^{\alpha, p}\left(\mathbb{R}^{n}\right)$, we refer to the references $[\mathbf{2}],[\mathbf{3}],[\mathbf{6}],[\mathbf{1 0}],[\mathbf{1 1}]$ and $[\mathbf{1 2}]$. It is obvious that $\dot{K}_{q}^{\alpha / q, q}\left(\mathbb{R}^{n}\right)=$ $L^{q}\left(\mathbb{R}^{n},|x|^{\alpha} d x\right)$. Our main results in this paper can be stated as follows.

Theorem 1. Let $1<q<\infty, 0<p \leq \infty,-n<\beta_{1}<\beta_{2}<n(q-1)$, $T$ be a sublinear integral operator which is bounded on $L^{q}\left(\mathbb{R}^{n}\right)$ and satisfies

$$
|T f(x)| \leq \int_{\mathbb{R}^{n}}|b(x)-b(y)|^{m}|K(x, y) f(y)| d y,
$$

where $m$ is a positive integer and $b \in \mathrm{BMO}\left(\mathbb{R}^{n}\right), K(x, y)$ is a function on $\mathbb{R}^{n} \times \mathbb{R}^{n} \backslash\{(x, y): x \neq y\}$. For $s \geq 1$, set $\Phi_{s}(t)=t \log ^{s}(2+t)$. Suppose that for any $0<r<\infty$ and $\beta_{1}<\beta<\beta_{2}$, the operator

$$
U_{m, r} f(x)=\int_{r<|x-y| \leq 2 r} \Phi_{m}\left(r^{n}|K(x, y)|\right)|f(y)| d y
$$

is bounded on $L^{q}\left(\mathbb{R}^{n},|x|^{\beta} d x\right)$ with bound $B r^{n}$ and $B$ is independent of $r$. Then the operator $T$ is bounded on the Herz space $\dot{K}_{q}^{\alpha, p}\left(\mathbb{R}^{n}\right)$ provided that $\beta_{1} / q<\alpha<\beta_{2} / q$.

Theorem 2. Let $1<q<\infty, 0<p \leq \infty,-n<\beta_{1}<\beta_{2}<n(q-1)$, $T$ be a sublinear integral operator which is bounded on $L^{q}\left(\mathbb{R}^{n}\right)$ and satisfies

$$
|T f(x)| \leq \int_{\mathbb{R}^{n}}|K(x, y)| \frac{\left|R_{m+1}(A ; x, y)\right|}{|x-y|^{n+m}}|f(y)| d y,
$$

where $K(x, y)$ is a function on $\mathbb{R}^{n} \times \mathbb{R}^{n} \backslash\{(x, y): x \neq y\}, m$ is a positive integer, $A$ has derivatives of order $m$ in $\operatorname{BMO}\left(\mathbb{R}^{n}\right), R_{m+1}$ is the $(m+1)$-th order Taylor series remainder of $A$ at $x$ expanded about $y$, that is,

$$
R_{m+1}(A ; x, y)=A(x)-\sum_{|\mu| \leq m} D^{\mu} A(y)(x-y)^{\mu} .
$$

Suppose that for any $0<r<\infty$ and $\beta_{1}<\beta<\beta_{2}$, the operator $U_{1, r}$ defined by (3) is bounded on $L^{q}\left(\mathbb{R}^{n},|x|^{\beta} d x\right)$ with bound $B r^{n}$ and $B$ is independent of $r$. Then the operator $T$ is bounded on the Herz space $\dot{K}_{q}^{\alpha, p}\left(\mathbb{R}^{n}\right)$ provided that $\beta_{1} / q<\alpha<\beta_{2} / q$.

To prove Theorem 1 and Theorem 2, we will use 
Theorem 3. Let $1<q<\infty, 0<p \leq \infty, \beta_{1}, \beta_{2} \in \mathbb{R}$ and $\beta_{1}<\beta_{2}$, $T$ be a sublinear integral operator which is bounded on $L^{q}\left(\mathbb{R}^{n}\right)$ and satisfies

$$
|T f(x)| \leq \int_{\mathbb{R}^{n}}|K(x, y) f(y)| d y .
$$

Suppose that for any $0<r<\infty$ and $\beta_{1}<\beta<\beta_{2}$, the operator

$$
T_{r} f(x)=\int_{r<|x-y| \leq 2 r}|K(x, y) f(y)| d y
$$

is bounded on $L^{q}\left(\mathbb{R}^{n},|x|^{\beta} d x\right)$ with bound independent of $r$. Then $T$ is bounded on the Herz space $\dot{K}_{q}^{\alpha, p}\left(\mathbb{R}^{n}\right)$ provided that $\beta_{1} / q<\alpha<\beta_{2} / q$.

Remark. Theorem 3 has independent interest and is more general and suitable for many operators in harmonic analysis, but it should be pointed out that the main idea in the proof of this theorem comes from the paper $[\mathbf{1 0}]$.

Throughout this paper, $C$ denotes the constants that are independent of the main parameters involved but whose value may differ from line to line, $A_{q}$ denotes the weight function class of Muckenhoupt (see [16, Chapter V] for definition and the properties of $A_{q}$ ). For a cube $I$, let $I^{*}=4 n I$. For a locally integrable function $f$, a real number $s \geq 1$, a cube $I$ and a nonnegative weighted function $w$, define

$$
\|f\|_{L(\log L)^{s} ; I, w}=\inf \left\{\lambda>0: \frac{1}{w(I)} \int_{I} \frac{|f(y)|}{\lambda} \log ^{s}\left(2+\frac{|f(y)|}{\lambda}\right) w(y) d y \leq 1\right\}
$$

and

$\|f\|_{\exp (L)^{1 / s ; I, w}}=\inf \left\{\lambda>0: \frac{1}{w(I)} \int_{I} \exp \left(\frac{|f(y)|}{\lambda}\right)^{1 / s} w(y) d y \leq 2\right\}$,

where $w(I)=\int_{I} w(y) d y$. Since that $\Phi_{s}(t)=t \log ^{s}(2+t)$ is a Young function on $[0, \infty)$ and its complementary Young function is $\Psi_{s}(t) \approx$ $\exp t^{1 / s}$, the generalized Hölder inequality

$$
\frac{1}{w(I)} \int_{I}|f(y) h(y)| d y \leq C\|f\|_{L(\log L)^{s} ; I, w}\|h\|_{\exp (L)^{1 / s} ; I, w}
$$

holds for locally integrable functions $f$ and $h$, see $[\mathbf{1}$, Chapter 8$]$ or $[\mathbf{1 3}$, p. 168] for details. 


\section{Proof of Theorems}

We begin with some preliminary lemmas.

Lemma 1 (see $[4])$. Let $A(x)$ be a function on $\mathbb{R}^{n}$ with derivatives of order $m$ in $L^{s}\left(\mathbb{R}^{n}\right)$ for some $n<s \leq \infty$. Then

$\left|R_{m}(A ; x, y)\right| \leq C_{m, n}|x-y|^{m} \sum_{|\mu|=m}\left(\frac{1}{|\tilde{Q}(x, y)|} \int_{\tilde{Q}(x, y)}\left|D^{\mu} A(z)\right|^{s} d z\right)^{1 / s}$,

where $\widetilde{Q}(x, y)$ is the cube centered at $x$ and having diameter $5 \sqrt{n}|x-y|$.

Lemma 2. Let $m$ be a positive integer and $b \in \operatorname{BMO}\left(\mathbb{R}^{n}\right), 0<r<\infty$ and $1<q<\infty, K(x, y)$ be defined on $\mathbb{R}^{n} \times \mathbb{R}^{n}$. Suppose that for some $w \in A_{q}$, the operator

$$
V f(x)=\int_{\mathbb{R}^{n}} \Phi_{m}\left(r^{n}|K(x, y)|\right) f(y) d y
$$

is bounded on $L^{q}\left(\mathbb{R}^{n}, w(x) d x\right)$ with bound $C r^{n}$. Then there exists some constant $C=C(n, m, q)$ such that for each integer $l$ with $0 \leq l \leq m$ and each cube $Q$ with side length $r$, the operator

$$
S_{l} f(x)=\int_{\mathbb{R}^{n}} \Phi_{l}\left(r^{n}|K(x, y)|\right) f(y) d y
$$

satisfies

$$
\left\|\left(S_{l} f\right)^{q}\right\|_{L(\log L)^{(m-l) q}, Q^{*}, w} \leq C r^{n q} w(Q)^{-1}\|f\|_{L^{q}\left(\mathbb{R}^{n}, w(x) d x\right)}^{q}
$$

provided that supp $f \subset Q$.

Proof: Without loss of generality, we may assume that $\|f\|_{1}=1$. Note that

$$
\begin{gathered}
\inf \left\{\lambda>0 ; \frac{1}{w\left(Q^{*}\right)} \int_{Q^{*}} \Phi_{(m-l) q}\left(\frac{\left|S_{l} f(x)\right|^{q}}{\lambda}\right) w(x) d x \leq 1\right\} \\
\leq\left(\inf \left\{\lambda>0 ; \frac{q^{m q}}{w\left(Q^{*}\right)} \int_{Q^{*}}\left(\Phi_{m-l}\left(\frac{\left|S_{l} f(x)\right|}{\lambda}\right)\right)^{q} w(x) d x \leq 1\right\}\right)^{q} \\
\leq q^{q m}\left(\inf \left\{\lambda>0 ; \frac{1}{w\left(Q^{*}\right)} \int_{Q^{*}}\left(\Phi_{m-l}\left(\frac{\left|S_{l} f(x)\right|}{\lambda}\right)\right)^{q} w(x) d x \leq 1\right\}\right)^{q} .
\end{gathered}
$$


Obviously, there exists a positive constant $C(m)$ such that for any $t>0$, $\Phi_{m-l}\left(\Phi_{l}(t)\right) \leq C(m) \Phi_{m}(t)$. By the Jensen inequality, we have

$$
\Phi_{m-l}\left(\left|S_{l} f(x)\right|\right) \leq \int_{Q} \Phi_{m-l}\left(\Phi_{l}\left(r^{n}|K(x, y)|\right)\right)|f(y)| d y \leq C V(|f|)(x) .
$$

Thus,

$$
\int_{\mathbb{R}^{n}}\left(\Phi_{m-l}\left(\left|S_{l} f(x)\right|\right)\right)^{q} w(x) d x \leq C r^{n q}\|f\|_{L^{q}\left(\mathbb{R}^{n}, w(x) d x\right)}^{q} .
$$

Let $q^{\prime}=q /(q-1)$ and $\widetilde{w}(x)=w(x)^{-q^{\prime} / q}$. Recall that $\|f\|_{1}=1$, it follows that

$$
1=\|f\|_{1}^{q} \leq\|f\|_{L^{q}\left(\mathbb{R}^{n}, w(x) d x\right)}^{q} \widetilde{w}(Q)^{q / q^{\prime}},
$$

which together with the fact $w \in A_{q}$ implies

$$
\frac{r^{n q}\|f\|_{L^{q}\left(\mathbb{R}^{n}, w(x) d x\right)}^{q}}{w(Q)} \geq \frac{r^{n q}}{w(Q) \widetilde{w}(Q)^{q / q^{\prime}}} \geq C .
$$

Choose $\lambda_{0}=r^{n}\|f\|_{L^{q}\left(\mathbb{R}^{n}, w(x) d x\right)} w(Q)^{-1 / q}$. Trivial computation gives that

$$
\begin{aligned}
\frac{1}{w\left(Q^{*}\right)} \int_{Q^{*}}\left(\Phi_{m-l}\right. & \left.\left(\frac{\left|S_{l} f(x)\right|}{\lambda_{0}}\right)\right)^{q} w(x) d x \\
& \leq \frac{C}{w\left(Q^{*}\right) \lambda_{0}^{q}} \int_{Q^{*}}\left(\Phi_{m-l}\left(\left|S_{l} f(x)\right|\right)\right)^{q} w(x) d x \leq C,
\end{aligned}
$$

and so

$$
\inf \left\{\lambda>0 ; \frac{1}{w\left(Q^{*}\right)} \int_{Q^{*}}\left(\Phi_{m-l}\left(\frac{\left|S_{l} f(x)\right|}{\lambda}\right)\right)^{q} w(x) d x \leq 1\right\} \leq C \lambda_{0} .
$$

This leads to the desired estimate.

Proof of Theorem 3: We only consider the case of $0<p<\infty$, the proof for the case $p=\infty$ is very similar. Set $\alpha_{1}, \alpha_{2} \in \mathbb{R}$ such that $\beta_{1} / q<$ $\alpha_{1} / q<\alpha<\alpha_{2} / q<\beta_{2} / q$. Write

$$
f=\sum_{j=-\infty}^{\infty} f \chi_{j}=\sum_{j=-\infty}^{\infty} f_{j} .
$$


The $L^{q}\left(\mathbb{R}^{n}\right)$ boundedness of $T$ gives that

$$
\begin{aligned}
\|T f\|_{\dot{K}_{q}^{\alpha, p}\left(\mathbb{R}^{n}\right)}^{p} \leq & C \sum_{k=-\infty}^{\infty} 2^{k \alpha p}\left\|\chi_{k} T\left(\sum_{j=k-2}^{k+2} f_{j}\right)\right\|_{L^{q}\left(\mathbb{R}^{n}\right)}^{p} \\
& +\sum_{k=-\infty}^{\infty} 2^{k \alpha p}\left\|\chi_{k} T\left(\sum_{j=-\infty}^{k-3} f_{j}\right)\right\|_{L^{q}\left(\mathbb{R}^{n}\right)}^{p} \\
& +\sum_{k=-\infty}^{\infty} 2^{k \alpha p}\left\|\chi_{k} T\left(\sum_{j=k+3}^{\infty} f_{j}\right)\right\|_{L^{q}\left(\mathbb{R}^{n}\right)}^{p} \\
\leq & C \sum_{k=-\infty}^{\infty} 2^{k \alpha p} \sum_{j=k-2}^{k+2}\left\|f_{j}\right\|_{L^{q}\left(\mathbb{R}^{n}\right)}^{p} \\
& +\sum_{k=-\infty}^{\infty} 2^{k\left(\alpha-\alpha_{2} / q\right) p}\left(\sum_{j=-\infty}^{k-3}\left\|\chi_{k} T f_{j}\right\|_{L^{q}\left(\mathbb{R}^{n},|x|^{\alpha_{2}} d x\right)}\right)^{p} \\
& +\sum_{k=-\infty}^{\infty} 2^{k\left(\alpha-\alpha_{1} / q\right) p}\left(\sum_{j=k+3}^{\infty}\left\|\chi_{k} T f_{j}\right\|_{L^{q}\left(\mathbb{R}^{n},|x|^{\alpha_{1}} d x\right)}\right)^{p} \\
\leq & C\|f\|_{\dot{K}_{q}^{\alpha, p}\left(\mathbb{R}^{n}\right)}^{p} \\
& +C \sum_{k=-\infty}^{\infty} 2^{k\left(\alpha-\alpha_{2} / q\right) p}\left(\sum_{j=-\infty}^{k-3}\left\|\chi_{k} T f_{j}\right\|_{L^{q}\left(\mathbb{R}^{n},|x|^{\alpha_{2}} d x\right)}\right)^{p} \\
& +C \sum_{k=-\infty}^{\infty} 2^{k\left(\alpha-\alpha_{1} / q\right) p}\left(\sum_{j=k+3}^{\infty}\left\|\chi_{k} T f_{j}\right\|_{L^{q}\left(\mathbb{R}^{n},|x|^{\alpha_{1}} d x\right)}\right)^{p} .
\end{aligned}
$$

Obviously, if $k \in \mathbb{Z}$ and $j \leq k-3$, then

$$
\left|\chi_{k}(x) T f_{j}(x)\right| \leq \int_{2^{k-2}<|x-y| \leq 2^{k+1}}\left|K(x-y) f_{j}(y)\right| d y .
$$

Similary, for $k \in \mathbb{Z}$ and $j \geq k+3$,

$$
\left|\chi_{k}(x) T f_{j}(x)\right| \leq \int_{2^{j-2}<|x-y| \leq 2^{j+1}}\left|K(x, y) f_{j}(y)\right| d y .
$$


Our hypothesis now says that

$$
\begin{aligned}
& \sum_{k=-\infty}^{\infty} 2^{k\left(\alpha-\alpha_{2} / q\right) p}\left(\sum_{j=-\infty}^{k-3}\left\|\chi_{k} T f_{j}\right\|_{L^{q}\left(\mathbb{R}^{n},|x|^{\alpha_{2}} d x\right)}\right)^{p} \\
+ & \sum_{k=-\infty}^{\infty} 2^{k\left(\alpha-\alpha_{1} / q\right) p}\left(\sum_{j=k+3}^{\infty}\left\|\chi_{k} T f_{j}\right\|_{L^{q}\left(\mathbb{R}^{n},|x|^{\alpha_{1}} d x\right)}\right)^{p} \\
\leq & \left.C \sum_{k=-\infty}^{\infty} 2^{k\left(\alpha-\alpha_{2} / q\right) p}\left(\sum_{j=-\infty}^{k-3}\left\|f_{j}\right\|_{L^{q}\left(\mathbb{R}^{n},|x|^{\alpha_{2}} d x\right)}\right)^{p}\right)^{p} \\
+ & C \sum_{k=-\infty}^{\infty} 2^{k\left(\alpha-\alpha_{1} / q\right) p}\left(\sum_{j=k+3}^{\infty}\left\|f_{j}\right\|_{L^{q}\left(\mathbb{R}^{n},|x|^{\alpha_{1}}\right) d x}\right)^{p} \\
\leq & C \sum_{k=-\infty}^{\infty}\left(\sum_{j=-\infty}^{k-3} 2^{j \alpha}\left\|f_{j}\right\|_{L^{q}\left(\mathbb{R}^{n}\right)} 2^{(k-j)\left(\alpha-\alpha_{2} / q\right)}\right)^{p} \\
+ & C \sum_{k=-\infty}^{\infty}\left(\sum_{j=k+3}^{\infty} 2^{j \alpha}\left\|f_{j}\right\|_{L^{q}\left(\mathbb{R}^{n}\right)} 2^{(k-j)\left(\alpha-\alpha_{1} / q\right)}\right)^{p}
\end{aligned}
$$

For the case of $0<p \leq 1$, we have

$$
\begin{aligned}
\|T f\|_{\dot{K}_{q}^{\alpha, p}\left(\mathbb{R}^{n}\right)} \leq & C\|f\|_{\dot{K}_{q}^{\alpha, p}\left(\mathbb{R}^{n}\right)} \\
& +C \sum_{k=-\infty}^{\infty} \sum_{j=-\infty}^{k-3} 2^{j \alpha p}\left\|f_{j}\right\|_{L^{q}\left(\mathbb{R}^{n}\right)}^{p} 2^{(k-j)\left(\alpha-\alpha_{2} / q\right) p} \\
& +C \sum_{k=-\infty}^{\infty} \sum_{j=k+3}^{\infty} 2^{j \alpha p}\left\|f_{j}\right\|_{L^{q}\left(\mathbb{R}^{n}\right)}^{p} 2^{(k-j)\left(\alpha-\alpha_{1} / q\right) p} \\
= & C\|f\|_{\dot{K}_{q}^{\alpha, p}\left(\mathbb{R}^{n}\right)}^{p} \\
& +\sum_{j=-\infty}^{\infty} 2^{j \alpha p}\left\|f_{j}\right\|_{L^{q}\left(\mathbb{R}^{n}\right)}^{p} \sum_{k=j+3}^{\infty} 2^{(k-j)\left(\alpha-\alpha_{2} / q\right) p} \\
& +C \sum_{j=-\infty}^{\infty} 2^{j \alpha p}\left\|f_{j}\right\|_{L^{q}\left(\mathbb{R}^{n}\right)}^{p} \sum_{k=-\infty}^{j-3} 2^{(k-j)\left(\alpha-\alpha_{2} / q\right) p} \\
= & C\|f\|_{\dot{K}_{q}^{\alpha, p}\left(\mathbb{R}^{n}\right)}^{p}
\end{aligned}
$$


On the other hand, if $1<p<\infty$, it follows from the Hölder inequality that

$$
\begin{aligned}
\|T f\|_{\dot{K}_{q}^{\alpha, p}\left(\mathbb{R}^{n}\right)}^{p} \leq & C\|f\|_{\dot{K}_{q}^{\alpha, p}\left(\mathbb{R}^{n}\right)}^{p} \\
& +C \sum_{k=-\infty}^{\infty} \sum_{j=-\infty}^{k-3} 2^{j \alpha p}\left\|f_{j}\right\|_{L^{q}\left(\mathbb{R}^{n}\right)}^{p} 2^{(k-j)\left(\alpha-\alpha_{2} / q\right) p / 2} \\
& \times\left(\sum_{j=-\infty}^{k-3} 2^{(k-j)\left(\alpha-\alpha_{2} / q\right) p^{\prime} / 2}\right)^{p / p^{\prime}} \\
& +C \sum_{k=-\infty}^{\infty} \sum_{j=k+3}^{\infty} 2^{j \alpha p}\left\|f_{j}\right\|_{L^{q}\left(\mathbb{R}^{n}\right)}^{p} 2^{(k-j)\left(\alpha-\alpha_{1} / q\right) p / 2} \\
\leq & C\|f\|_{\dot{K}_{q}^{\alpha, p}\left(\mathbb{R}^{n}\right)}^{p} \times\left(\sum_{j=k+3}^{\infty} 2^{(k-j)\left(\alpha-\alpha_{1} / q\right) p^{\prime} / 2}\right)^{p / p^{\prime}} \\
& +C \sum_{j=-\infty}^{\infty} 2^{j \alpha p}\left\|f_{j}\right\|_{L^{q}\left(\mathbb{R}^{n}\right)}^{p} \sum_{k=j+3}^{\infty} 2^{(k-j)\left(\alpha-\alpha_{2} / q\right) p / 2} \\
& +C \sum_{j=-\infty}^{\infty} 2^{j \alpha p}\left\|f_{j}\right\|_{L^{q}\left(\mathbb{R}^{n}\right)}^{p} \sum_{k=-\infty}^{j-3} 2^{(k-j)\left(\alpha-\alpha_{1} / q\right) p / 2} \\
\leq & C\|f\|_{\dot{K}_{q}^{\alpha, p}\left(\mathbb{R}^{n}\right)}^{\cdot}
\end{aligned}
$$

This completes the proof of Theorem 3.

Theorem 1 is an easy consequence of Theorem 3 and the following Lemma 3.

Lemma 3. Let $m$ be a positive integer and $b \in \operatorname{BMO}\left(\mathbb{R}^{n}\right), 0<r<\infty$ and $1<q<\infty, K(x, y)$ be defined on $\mathbb{R}^{n} \times \mathbb{R}^{n}$. Suppose that for some $w \in A_{q}$, the operator

$$
U_{m, r} f(x)=\int_{r<|x-y| \leq 2 r} \Phi_{m}\left(r^{n}|K(x, y)|\right) f(y) d y
$$

is bounded on $L^{q}\left(\mathbb{R}^{n}, w(x) d x\right)$ with bound $B r^{n}$. Then the commutator

$$
S_{r ; b, m} f(x)=\int_{r<|x-y| \leq 2 r}|K(x, y)||b(x)-b(y)|^{m}|f(y)| d y
$$

is bounded on $L^{q}\left(\mathbb{R}^{n}, w(x) d x\right)$ with bound $C(n, m, q, B)\|b\|_{\mathrm{BMO}\left(\mathbb{R}^{n}\right)}^{m}$. 
Proof: Without loss of generality, we may assume that $\|b\|_{\mathrm{BMO}\left(\mathbb{R}^{n}\right)}=1$. Write $\mathbb{R}^{n}=\bigcup_{j \in \mathbb{Z}} Q_{j}$, where each $Q_{j}$ is a cube with side length $r$, and these cubes have disjoint interiors. Let $f_{j}$ be the restriction of $f$ on $Q_{j}$. Then

$$
f(x)=\sum_{j \in \mathbb{Z}} f_{j}(x), \text { a.e. } x \in \mathbb{R}^{n} .
$$

Observe that the support of $S_{r ; b, m} f_{j}$ is contained in $Q_{j}^{*}$, and that the supports of various terms $S_{r ; b, m} f_{j}$ have bounded overlaps. So we have

$$
\left\|S_{r ; b, m} f\right\|_{L^{q}\left(\mathbb{R}^{n}, w(x) d x\right)}^{q} \leq C \sum_{j \in \mathbb{Z}}\left\|S_{r ; b, m} f_{j}\right\|_{L^{q}\left(\mathbb{R}^{n}, w(x) d x\right)}^{q},
$$

where $C$ is a positive constant which is independent of $f$ and $j$. Thus we may assume that $\operatorname{supp} f \subset Q$ for some cube $Q$ with side length $r$. Let $\varphi \in C_{0}^{\infty}\left(\mathbb{R}^{n}\right), 0 \leq \varphi \leq 1, \varphi$ is identically one on $Q^{*}$ and vanishes outside $Q^{* *}$. Let $\widetilde{b}(x)=\left(b(x)-m_{Q^{*}}(b)\right) \varphi(x)$, where $m_{Q^{*}}(b)$ denotes the mean value of $b$ on $Q^{*}$. Define the operator $\widetilde{S}_{r}$ by

$$
\widetilde{S}_{r} h(x)=r^{n} \int_{r<|x-y| \leq 2 r}|K(x, y) h(y)| d y .
$$

Write

$$
S_{r ; b, m} f(x) \leq\left.\sum_{l=0}^{m} C_{m}^{l} \widetilde{b}(x)\right|^{l} \widetilde{S}_{r}\left(\widetilde{b}^{m-l} f\right)(x) r^{-n} .
$$

It is enough to show that for each $l, 0 \leq l \leq m$,

$$
\left\|\widetilde{b}^{l} \widetilde{S}_{r}\left(\widetilde{b}^{m-l} f\right)\right\|_{L^{q}\left(\mathbb{R}^{n}, w(x) d x\right)}^{q} \leq C\|f\|_{L^{q}\left(\mathbb{R}^{n}, w(x) d x\right)}^{q} r^{n q} .
$$

By the generalized Hölder inequality and the well-known John-Nirenberg inequality,

$$
\begin{aligned}
& \left\|\widetilde{b}^{l} \widetilde{S}_{r}\left(\widetilde{b}^{m-l} f\right)\right\|_{L^{q}\left(\mathbb{R}^{n}, w(x) d x\right)}^{q} \\
& \quad \leq C w\left(Q^{*}\right)\left\|\widetilde{b}^{l q}\right\|_{\exp (L)^{1 /(l q)}, Q^{*}, w}\left\|\left(\widetilde{S}_{r}\left(\widetilde{b}^{m-l} f\right)\right)^{q}\right\|_{L(\log L)^{l q}, Q^{*}, w} \\
& \quad \leq C w\left(Q^{*}\right)\left\|\left(\widetilde{S}_{r}\left(\widetilde{b}^{m-l} f\right)\right)^{q}\right\|_{L(\log L)^{l q}, Q^{*}, w} .
\end{aligned}
$$

As in the proof of Lemma 2, we have

$$
\begin{aligned}
& \left\|\left(\widetilde{S}_{r}\left(\widetilde{b}^{m-l} f\right)\right)^{q}\right\|_{L(\log L)^{l q}, Q^{*}, w} \\
\leq & C\left(\inf \left\{\lambda>0 ; \frac{1}{w\left(Q^{*}\right)} \int_{Q^{*}}\left(\Phi_{l}\left(\frac{\left|\widetilde{S}_{r}\left(\widetilde{b}^{m-l} f\right)(x)\right|}{\lambda}\right)\right)^{q} w(x) d x \leq 1\right\}\right)^{q} .
\end{aligned}
$$


Thus, the proof of the estimate (6) can be reduced to proving that

$$
\begin{array}{r}
\inf \left\{\lambda>0 ; \frac{1}{w\left(Q^{*}\right)} \int_{Q^{*}}\left(\Phi_{l}\left(\frac{\left|\widetilde{S}_{r}\left(\widetilde{b}^{m-l} f\right)(x)\right|}{\lambda}\right)\right)^{q} w(x) d x \leq 1\right\} \\
\leq C w(Q)^{-1 / q}\|f\|_{L^{q}\left(\mathbb{R}^{n}, w(x) d x\right)}|Q| .
\end{array}
$$

We claim that

$$
\begin{aligned}
& \frac{1}{w\left(Q^{*}\right)} \int_{Q^{*}}\left(\Phi_{l}\left(\frac{\left|\widetilde{S}_{r}\left(\widetilde{b}^{m-l} f\right)(x)\right|}{\left\|\widetilde{b}^{m-l} f\right\|_{1}}\right)\right)^{q} w(x) d x \\
& \quad \leq C w(Q)^{-1}|Q|^{q}\|f\|_{L^{q}\left(\mathbb{R}^{n}, w(x) d x\right)}^{q}\left\|\widetilde{b}^{m-l} f\right\|_{1}^{-q} .
\end{aligned}
$$

In fact, by the Jensen inequality,

$$
\Phi_{l}\left(\frac{\left|\widetilde{S}_{r}\left(\widetilde{b}^{m-l} f\right)(x)\right|}{\left\|\widetilde{b}^{m-l} f\right\|_{1}}\right) \leq \int_{r<|x-y| \leq 2 r} \Phi_{l}\left(r^{n}|K(x, y)|\right) \frac{\left|\widetilde{b}^{m-l}(y) f(y)\right|}{\left\|\widetilde{b}^{m-l} f\right\|_{1}} d y
$$

Let

$$
U_{l} h(x)=\int_{r<|x-y| \leq 2 r} \Phi_{l}\left(r^{n} \mid K(x, y \mid) h(y) d y .\right.
$$

Denote by $U_{l}^{*}$ the dual operator of $U_{l}$, that is

$$
U_{l}^{*} h(y)=\int_{r<|x-y| \leq 2 r} \Phi_{l}\left(r^{n}|K(x, y)|\right) h(x) d x .
$$

For each fixed $g \in L^{q^{\prime}}\left(\mathbb{R}^{n}, \widetilde{w}(x) d x\right)$, supp $g \subset Q^{*}$ and $\|g\|_{L^{q^{\prime}}\left(\mathbb{R}^{n}, \tilde{w}(x) d x\right)} \leq$ 1 , the inequality (7) says that

$$
\begin{aligned}
& \int_{\mathbb{R}^{n}} g(x) \Phi_{l}\left(\frac{\left|\widetilde{S}_{r}\left(\widetilde{b}^{m-l} f\right)(x)\right|}{\left\|\widetilde{b}^{m-l} f\right\|_{1}}\right) \\
\leq & \int_{\mathbb{R}^{n}} \frac{\left|f(y) \| \widetilde{b}^{m-l}(y)\right|}{\left\|\widetilde{b}^{m-l} f\right\|_{1}} \int_{r<|x-y| \leq 2 r} \Phi_{l}\left(r^{n}|K(x, y)|\right) g(x) d x d y \\
\leq & \|f\|_{L^{q}\left(\mathbb{R}^{n}, w(x) d x\right)}\left\|\widetilde{b}^{m-l} U_{l}^{*} g\right\|_{L^{q^{\prime}}\left(\mathbb{R}^{n}, \tilde{w}(x) d x\right)}\left\|\widetilde{b}^{m-l} f\right\|_{1}^{-1} .
\end{aligned}
$$

Applying the generalized Hölder inequality and the John-Nirenberg inequality again, we have

$$
\left\|\widetilde{b}^{m-l} U_{l}^{*} g\right\|_{q^{\prime}, \tilde{w}}^{q^{\prime}} \leq C \widetilde{w}(Q)\left\|\left(U_{l}^{*} g\right)^{q^{\prime}}\right\|_{L(\log L)^{(m-l) q^{\prime}, Q^{* *}, \tilde{w}}} .
$$


A standard duality arguement states that $U_{l}^{*}$ is bounded on $L^{q^{\prime}}\left(\mathbb{R}^{n}, \widetilde{w}(x) d x\right)$. This via Lemma 2 yields

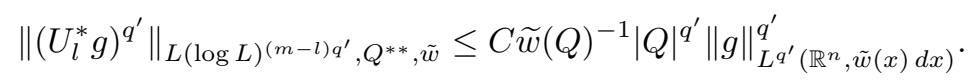

We thus obtain that

$$
\begin{aligned}
& \left\|\Phi_{l}\left(\widetilde{S}_{r}\left(\widetilde{b}^{m-l} f /\left\|\widetilde{b}^{m-l} f\right\|_{1}\right)\right)\right\|_{L^{q}\left(\mathbb{R}^{n}, w(x) d x\right)}
\end{aligned}
$$

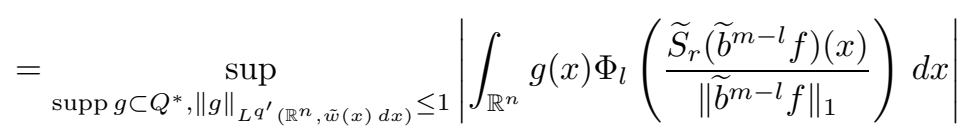

$$
\begin{aligned}
& \leq C\left\|\widetilde{b}^{m-l} f\right\|_{1}^{-1}\|f\|_{L^{q}\left(\mathbb{R}^{n}, w(x) d x\right)}|Q|,
\end{aligned}
$$

which leads to our claim.

We can now conclude the proof of the inequality (6). Note that $\widetilde{w} \in$ $A_{q^{\prime}}$, we can choose $r>1$ such that for any cube $I$,

$$
\int_{I} \widetilde{w}(x)^{r} d x \leq C|I|^{1-r}\left(\int_{I} \widetilde{w}(x) d x\right)^{r} .
$$

By the Hölder inequality, it follows that

$$
\begin{aligned}
&\left\|\widetilde{b}^{m-l} f\right\|_{1} \leq\|f\|_{L^{q}\left(\mathbb{R}^{n}, w(x) d x\right)}\left\|\widetilde{b}^{(m-l)}\right\|_{L^{q^{\prime}}\left(\mathbb{R}^{n}, \tilde{w}(x) d x\right)} \\
& \leq\|f\|_{L^{q}\left(\mathbb{R}^{n}, w(x) d x\right)}\left(\int_{Q^{* *}} \widetilde{b}(x)^{(m-l) q^{\prime} r^{\prime}} d x\right)^{1 /\left(q^{\prime} r^{\prime}\right)} \\
& \times\left(\int_{Q^{* *}} \widetilde{w}(x)^{r} d x\right)^{1 /\left(r q^{\prime}\right)} \\
& \leq C\|f\|_{L^{q}\left(\mathbb{R}^{n}, w(x) d x\right)} \widetilde{w}(Q)^{1 / q^{\prime}} .
\end{aligned}
$$

Therefore,

$\left\|\widetilde{b}^{m-l} f\right\|_{1}^{-q}\|f\|_{L^{q}\left(\mathbb{R}^{n}, w(x) d x\right)}^{q}|Q|^{q} w(Q)^{-1} \geq C|Q|^{q} w(Q)^{-1} \widetilde{w}(Q)^{-q / q^{\prime}} \geq C$.

Our claim together with the last estimate implies that

$\frac{1}{w\left(Q^{*}\right)} \int_{Q^{*}}\left(\Phi_{l}\left(\frac{\left.\widetilde{S}_{r}\left(\widetilde{b}^{m-l} f\right)(x) /\left\|\widetilde{b}^{m-l} f\right\|_{1}\right)}{\left\|\tilde{b}^{m-l} f\right\|_{1}^{-1} w(Q)^{-1 / q}|Q|\|f\|_{L^{q}\left(\mathbb{R}^{n}, w(x) d x\right)}}\right)\right)^{q} w(x) d x \leq C$, 
and

$$
\begin{array}{r}
\inf \left\{\lambda>0 ; \frac{1}{w\left(Q^{*}\right)} \int_{Q^{*}}\left(\Phi_{l}\left(\frac{\left.\widetilde{S}_{r}\left(\widetilde{b}^{m-l} f\right)(x)\right)}{\lambda}\right)\right)^{q} w(x) d x \leq 1\right\} \\
\leq C w(Q)^{-1 / q}\|f\|_{L^{q}\left(\mathbb{R}^{n}, w(x) d x\right)}|Q| .
\end{array}
$$

This finishes the proof of Lemma 3.

Now we turn our attention to the proof of Theorem 2, which can be obtained from Theorem 3 and the following Lemma 4 directly.

Lemma 4. Let $m$ be a positive integer and $1<q<\infty, A$ be a function on $\mathbb{R}^{n}$ with derivatives of order $m$ in $\operatorname{BMO}\left(\mathbb{R}^{n}\right), K(x, y)$ be defined on $\mathbb{R}^{n} \times \mathbb{R}^{n}$. Suppose that for some $w \in A_{q}$, the operator

$$
U_{1, r} f(x)=\int_{r<|x-y| \leq 2 r} \Phi_{1}\left(r^{n}|K(x, y)|\right) f(y) d y
$$

is bounded on $L^{q}\left(\mathbb{R}^{n}, w(x) d x\right)$ with bound $B r^{n}$. Then the operator

$$
V_{A ; r} f(x)=\int_{r<|x-y| \leq 2 r}|K(x, y)| \frac{\left|R_{m+1}(A ; x, y)\right|}{|x-y|^{m}}|f(y)| d y
$$

is bounded on $L^{q}\left(\mathbb{R}^{n}, w(x) d x\right)$ with bound $C(n, m, q, B) \sum_{|\mu|=m}\left\|D^{\mu} A\right\|_{\mathrm{BMO}\left(\mathbb{R}^{n}\right)}$.

Proof: As in the proof of Lemma 3, we may assume that supp $f \subset Q$ for some cube $Q$ with side length $r$. Without loss of generality, we may assume that $\sum_{|\mu|=m}\left\|D^{\mu} A\right\|_{\operatorname{BMO}\left(\mathbb{R}^{n}\right)}=1$. Set

$$
A^{Q}(y)=A(y)-\sum_{|\mu|=m} \frac{1}{\mu !} m_{Q}\left(D^{\mu} A\right) y^{\mu} .
$$

Note that for $x, y \in \mathbb{R}^{n}$ with $r<|x-y| \leq 2 r$ and $n<s<\infty$, it follows from Lemma 1 that

$$
\begin{aligned}
& \left|R_{m}\left(A^{Q} ; x, y\right)\right| \\
& \quad \leq C|x-y|^{m} \sum_{|\mu|=m}\left(\frac{1}{|\tilde{Q}(x, y)|} \int_{\tilde{Q}(x, y)}\left|D^{\mu} A(z)-m_{Q}\left(D^{\mu} A\right)\right|^{s} d z\right)^{1 / s} \\
& \quad \leq C|x-y|^{m} .
\end{aligned}
$$


Therefore,

$$
\begin{aligned}
V_{A ; r} f(x)= & \int_{r<|x-y| \leq 2 r}|K(x, y)| \frac{\left|R_{m+1}\left(A^{Q} ; x, y\right)\right|}{|x-y|^{m}}|f(y)| d y \\
\leq & C \int_{r<|x-y| \leq 2 r}|K(x-y) f(y)| d y \\
& +C \sum_{|\mu|=m} \int_{r<|x-y| \leq 2 r}|K(x-y)|\left|D^{\mu} A(y)-m_{Q}\left(D^{\mu} A\right)\right||f(y)| d y .
\end{aligned}
$$

By the estimate (6) (with $m=1$ and $l=0$ ) in the proof of Lemma 3, we finally obtain

$$
\left\|V_{A, r} f\right\|_{L^{q}\left(\mathbb{R}^{n}, w(x) d x\right)} \leq C B\|f\|_{L^{q}\left(\mathbb{R}^{n}, w(x) d x\right)} .
$$

\section{Some applications}

This section is devoted to some applications of our Theorem 1 and Theorem 2. We begin with the commutator of homogeneous singular integral operator. Note that $\Omega \in L(\log L)^{m}\left(S^{n-1}\right)$ is equivalent to that $\Phi_{m}(|\Omega|) \in L^{1}\left(S^{n-1}\right)$ and in this case the operator

$$
W_{m, r} f(x)=r^{-n} \int_{r<|x-y| \leq 2 r} \Phi_{m}(|\Omega(x-y)|) f(y) d y
$$

is bounded on $L^{q}\left(\mathbb{R}^{n},|x|^{\alpha} d x\right)$ for all $1<q<\infty$ and $-1<\alpha<q-1$ (see [5, p. 874]). This together with Theorem 2 tells us that

Corollary 1. Let $1<q<\infty, 0<p \leq \infty, T$ be a sublinear operator which satisfies the size condition

$$
|T f(x)| \leq \int_{\mathbb{R}^{n}}|b(x)-b(y)|^{m} \frac{|\Omega(x-y)|}{|x-y|^{n}}|f(y)| d y,
$$

where $m$ is a positive integer and $b \in \mathrm{BMO}\left(\mathbb{R}^{n}\right), \Omega$ is homogeneous of degree zero. If $T$ is bounded on $L^{q}\left(\mathbb{R}^{n}\right)$ and $\Omega \in L(\log L)^{m}\left(S^{n-1}\right)$, then $T$ is also bounded on $\dot{K}_{q}^{\alpha, p}$ with bound $C(n, m, p, \alpha)$ provided that $-1 / q<\alpha<(q-1) / q$.

For the operator $T_{b, m}$ defined by (1) in Section 1, we have

Corollary 2. Let $1<q<\infty, 0<p \leq \infty, \Omega$ be homogeneous of degree zero and have mean value zero. If $\Omega \in L(\log L)^{m+1}\left(S^{n-1}\right)$ for some positive integer $m$, then the operator $T_{b, m}$ defined by (1) is bounded on $\dot{K}_{q}^{\alpha, p}$ with bound $C(n, m, p, \alpha)$ provided that $-1 / q<\alpha<(q-1) / q$. 
Corollary 1 and Corollary 2 are new even for the the special case of $p=q$, i.e., the weighted Lebesgue spaces with power weights.

Now let us consider another class of nonstandard Calderón-Zygmund operators. We can easily obtain a general version similar to Corollary 1 , but for brevity we only consider the operator defined by

$$
T_{A} f(x)=\int_{\mathbb{R}^{n}} \frac{\Omega(x-y)}{|x-y|^{n+m}} R_{m+1}(A ; x, y) f(y) d y,
$$

where $\Omega$ is homogeneous of degree zero and has vanishing moment of order $m$, that is

$$
\int_{S^{n-1}} \Omega(\theta) \theta^{\mu} d \theta=0, \text { for any multi-index } \mu \text { with }|\mu|=m,
$$

$m$ is a positive integer, $A$ has derivatives of order $m$ in $\operatorname{BMO}\left(\mathbb{R}^{n}\right)$ and

$$
R_{m+1}(A ; x, y)=A(x)-\sum_{|\mu| \leq m} D^{\mu} A(y)(x-y)^{\mu} .
$$

We have proved in [7, pp. 68-69] that if $\Omega \in L(\log L)^{\gamma}\left(S^{n-1}\right)$ for some $\gamma>1$, then $K_{j}(x)=\frac{\Omega(x)}{|x|^{n}} \chi_{\left\{2^{j}<|x| \leq 2^{j+1}\right\}}(x)$ satisfies the Fourier transform estimate

$$
\left|\widehat{K}_{j}(\xi)\right| \leq C \min \left\{1, \log ^{-\gamma}\left(2+\left|2^{j} \xi\right|\right)\right\} .
$$

This together with Theorem 1 in [8] says that if $\Omega \in L(\log L)^{\gamma}\left(S^{n-1}\right)$ for some $\gamma>3$, then the operator $T_{A}$ is bounded on $L^{2}\left(\mathbb{R}^{n}\right)$. Thus by Theorem 3 , we can obtain

Corollary 3. Let $0<p \leq \infty, \Omega$ be homogeneous of degree zero and have vanishing moment of order $m, A$ have derivetives of order $m$ in $\operatorname{BMO}\left(\mathbb{R}^{n}\right)$. Suppose that $\Omega \in L(\log L)^{\gamma}\left(S^{n-1}\right)$ for some $\gamma>3$, then the operator defined by (8) is bounded on $\dot{K}_{2}^{\alpha, p}$ provided that $-1 / 2<\alpha<$ $1 / 2$.

Also, even for the special case of $p=2$, Corollary 3 is new.

Acknowledgement. This paper was finished while the author was visiting the Department of Mathematics and Statistics, University of Edinburgh. The author would like to thank the Department of Mathematics and Statistics for hospitality and Professor A. Carbery for his encouragement and helpful suggestions. The author would also like to thank professor Dachun Yang for some helpful discussions concerning the homogeneous Herz spaces. 


\section{References}

[1] R. A. Adams, "Sobolev spaces", Pure and Applied Mathematics 65, Academic Press, New York-London, 1975.

[2] A. Baernstein, II And E. T. SAwyer, Embedding and multiplier theorems for $H^{P}\left(\mathbb{R}^{n}\right)$, Mem. Amer. Math. Soc. 53(318) (1985), 82 pp.

[3] A. Beurling, Construction and analysis of some convolution algebras, Ann. Inst. Fourier (Grenoble) 14(2) (1964), 1-32.

[4] J. Cohen And J. Gosselin, A BMO estimate for multilinear singular integrals, Illinois J. Math. 30(3) (1986), 445-464.

[5] J. DuonndikoetxeA, Weighted norm inequalities for homogeneous singular integrals, Trans. Amer. Math. Soc. 336(2) (1993), 869-880.

[6] C. S. Herz, Lipschitz spaces and Bernstein's theorem on absolutely convergent Fourier transforms, J. Math. Mech. 18 (1968/69), $283-323$.

[7] G. Hu, $L^{2}\left(\mathbb{R}^{n}\right)$ boundedness for the commutators of convolution operators, Nagoya Math. J. 163 (2001), 55-70.

[8] G. Hu, $L^{2}\left(\mathbb{R}^{n}\right)$ boundedness for a class of multilinear singular integral operators, Acta Math. Sinica (N.S.) (to appear).

[9] G. Hu, $L^{p}\left(\mathbb{R}^{n}\right)$ boundedness for the commutators of homogeneous singular integral operators, Studia Math. 157 (2003), 13-27.

[10] G. Hu, S. LU AND D. YANG, Boundedness of rough singular integral operators on homogeneous Herz spaces, J. Austral. Math. Soc. Ser. A 66(2) (1999), 201-223.

[11] X. Li AND D. YANG, Boundedness of some sublinear operators on Herz spaces, Illinois J. Math. 40(3) (1996), 484-501.

[12] S. LU AND D. YANG, The continuity of commutators on Herz-type spaces, Michigan Math. J. 44(2) (1997), 255-281.

[13] C. PÉREz, Endpoint estimates for commutators of singular integral operators, J. Funct. Anal. 128(1) (1995), 163-185.

[14] F. Soria AND G. Weiss, A remark on singular integrals and power weights, Indiana Univ. Math. J. 43(1) (1994), 187-204.

[15] E. M. Stein, Note on singular integrals, Proc. Amer. Math. Soc. 8 (1957), 250-254.

[16] E. M. STEIN, "Harmonic analysis: real-variable methods, orthogonality, and oscillatory integrals", Princeton Mathematical Series 43, Monographs in Harmonic Analysis III, Princeton University Press, Princeton, NJ, 1993. 
Department of Applied Mathematics

University of Information Engineering

P. O. Box 1001-747

Zhengzhou 450002

People's Republic of China

E-mail address: huguoen@eyou.com

Primera versió rebuda el 19 de febrer de 2002, darrera versió rebuda el 23 de setembre de 2002. 